\title{
Four-Photon Interference with Asymmetric Beam Splitters
}

\author{
B. H. Liu, F. W. Sun, Y. X. Gong, Y. F. Huang, and G. C. Guo \\ Key Laboratory of Quantum Information, University of Science and Technology of China, \\ CAS, Hefei, 230026, the People's Republic of China \\ Z. Y. $\mathrm{Ou}^{*}$ \\ Department of Physics, Indiana University-Purdue University Indianapolis, \\ 402 N. Blackford Street, Indianapolis, IN 46202, USA
}

\begin{abstract}
Two experiments of four-photon interference are performed with two pairs of photons from parametric down-conversion with the help of asymmetric beam splitters. The first experiment is a generalization of the Hong-Ou-Mandel interference effect to two pairs of photons while the second one utilizes this effect to demonstrate a four-photon de Broglie wavelength of $\lambda / 4$ by projection measurement.
\end{abstract}

PACS numbers:

Multi-photon quantum interference plays a pivotal role in quantum information sciences. Although two-photon interference has been widely studied [1] and is applied to some quantum information protocols [2], quantum interference of more than two photons has only recently been the focus of research because of its role in the fundamental study of quantum nonlocality [3, 4] and the improvement in the precision phase measurement [5, 6, 7, [8, 9 ].

The most well-known two-photon interference is the Hong-Ou-Mandel effect [10], where two photons enter a symmetric beam splitter (50:50) from two sides respectively. Because of two-photon destructive interference, the probability for the two photons to exit at separate ports is zero. However, generalization to higher photon number is not straightforward. For example, for an input state of $\left|2_{a}, 2_{b}\right\rangle$, i.e., two from each side respectively (Fig.1a) to a symmetric beam splitter, there is a nonzero probability for the $\left|2_{A}, 2_{B}\right\rangle$ state at the output [1]], contrary to the two-photon counterpart.

Recently, however, Wang and Kobayashi [12] proposed a generalization of the Hong-Ou-Mandel effect to three photons with an asymmetric beam splitter $(T \neq R)$. With a state of $\left|2_{a}, 1_{b}\right\rangle$ input at a beam splitter with $T=2 R=2 / 3$, the probability is zero for the state $\left|2_{A}, 1_{B}\right\rangle$ in the output state, due to three-photon interference [12]. Sanaka et al. 13] demonstrated experimentally this three-photon Hong-Ou-Mandel effect.

Wang and Kobayashi went further and proposed a three-photon interferometer, which shows a three-photon de Broglie wavelength. The de Broglie wavelength of a multi-photon state is the equivalent matter wavelength for all the photons as one entity [14]. Thus the de Broglie wavelength for an $N$-photon state is simply $\lambda / N$ with $\lambda$ as the single-photon wavelength. It will show up in an interference fringe that is $N$ times finer than the regular single-photon fringe. It has applications in preci-

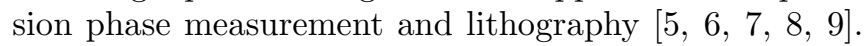
Liu et al. [15] implemented the scheme by Wang and Kobayashi and observed an interference pattern with the three-photon de Broglie wavelength.

In this Letter, we will generalize the idea of Wang and

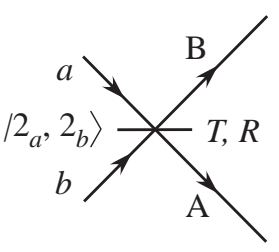

(a)

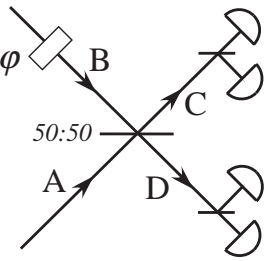

(b)
FIG. 1: (a) Hong-Ou-Mandel interferometer with asymmetric beam splitter and (b) formation of an interferometer for the de Broglie wavelength of four photons.

Kobayashi to the four-photon case. We send the input state of $\left|2_{a}, 2_{b}\right\rangle$ to an asymmetric beam splitter and observe a four-photon Hong-Ou-Mandel effect with proper adjustment of the transmissivity of the beam splitter. Then we form an interferometer and demonstrate the de Broglie wavelength for four photons.

When a state of $\left|2_{a}, 2_{b}\right\rangle$ enters an asymmetric beam splitter with $T \neq R$ (Fig.1a), the output state is [16]

$$
\begin{aligned}
\left|\Psi_{4}\right\rangle_{\text {out }}=\sqrt{6} & T R\left(\left|4_{A}, 0_{B}\right\rangle+\left|0_{A}, 4_{B}\right\rangle\right) \\
+ & \sqrt{6 T R}(T-R)\left(\left|3_{A}, 1_{B}\right\rangle-\left|1_{A}, 3_{B}\right\rangle\right) \\
& +\left[(T-R)^{2}-2 T R\right]\left|2_{A}, 2_{B}\right\rangle .
\end{aligned}
$$

For a symmetric beam splitter with $T=R=1 / 2$, we find a nonzero probability for the state $\left|2_{A}, 2_{B}\right\rangle$ in the output, as we discussed earlier. But when $(T-R)^{2}-2 T R=0$ or $T=(3 \pm \sqrt{3}) / 6, R=(3 \mp \sqrt{3}) / 6$, the $\left|2_{A}, 2_{B}\right\rangle$ term disappears from Eq.(11) and the probability of detecting two photons at each side is zero, i.e., $P_{4}\left(2_{A}, 2_{B}\right)=0$. Hence, we realize a generalized Hong-Ou-Mandel effect for two pairs of photons.

If we follow the outputs by another symmetric beam splitter as shown in Fig.1b, the $\left|3_{A}, 1_{B}\right\rangle$ and $\left|1_{A}, 3_{B}\right\rangle$ states in Eq.(1) will not contribute to the probability $P_{4}\left(2_{C}, 2_{D}\right)$ of detecting four photons with two at each side due to a two-photon Hong-Ou-Mandel effect. Since the $\left|2_{A}, 2_{B}\right\rangle$ state in Eq.(11) is cancelled out when $T=$ 


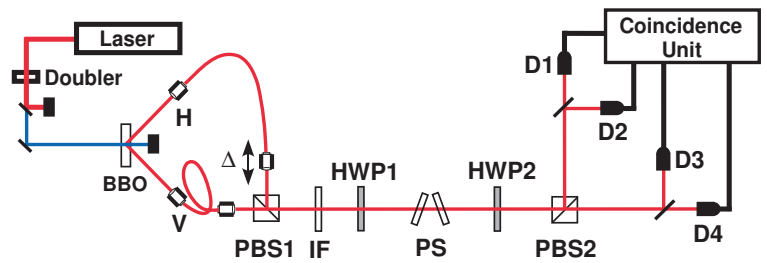

FIG. 2: The layout of the experiment. PBS: polarization beam splitter; HWP: half wave plate; PS: phase shifter; IF: interference filter; D1-D4: photo-detectors.

$(3 \pm \sqrt{3}) / 6, R=(3 \mp \sqrt{3}) / 6$, only the part of $|4,0\rangle+|0,4\rangle$ in Eq.(1) will contribute to $P_{4}\left(2_{C}, 2_{D}\right)$, leading to a fourphoton interference effect with

$$
P_{4}\left(2_{C}, 2_{D}\right) \propto 1+\cos 4 \varphi
$$

where $\varphi$ is the single-photon phase difference between $\mathrm{A}$ and $\mathrm{B}$. This can be easily confirmed by evaluating the four-photon detection probability $P_{4}\left(2_{C}, 2_{D}\right) \propto$ $\left\langle 2_{a}, 2_{b}\left|\hat{C}^{\dagger 2} \hat{D}^{\dagger 2} \hat{D}^{2} \hat{C}^{2}\right| 2_{a}, 2_{b}\right\rangle$ with

$$
\left\{\begin{array} { l } 
{ \hat { C } = ( \hat { A } + e ^ { j \varphi } \hat { B } ) / \sqrt { 2 } , } \\
{ \hat { D } = ( e ^ { j \varphi } \hat { B } - \hat { A } ) / \sqrt { 2 } , }
\end{array} \quad \left\{\begin{array}{l}
\hat{A}=\sqrt{T} \hat{a}+\sqrt{R} \hat{b}, \\
\hat{B}=\sqrt{T} \hat{b}-\sqrt{R} \hat{a},
\end{array}\right.\right.
$$

where $T=(3 \pm \sqrt{3}) / 6, R=1-T$.

Experimental implementation is shown in Fig.2, where the four-photon state of $\left|2_{a}, 2_{b}\right\rangle$ is produced from a type-II parametric down-conversion process pumped by 150 fsec frequency-doubled pulses from a mode-locked Ti:Sapphire laser operating at $780 \mathrm{~nm}$. The $2 \mathrm{~mm}$ long $\beta$-Barium Borate (BBO) crystal is so oriented that it produces two beam-like orthogonally polarized fields at the degenerate wavelength of $780 \mathrm{~nm}$ 17]. The horizontal $(\mathrm{H})$ and the vertical $(\mathrm{V})$ polarized fields are first coupled into single-mode fibers and are recombined with a polarization beam splitter (PBS1) into one beam before passing through an interference filter (IF) of $3 \mathrm{~nm}$ bandpass. The filtered field is then feeded into the fourphoton interferometer of Fig.1 But the beam splitters of Fig.1 are equivalently replaced by two polarization rotators (HWPs) and another polarization beam splitter (PBS2). Thus it is a polarization interferometer. A phase shifter (PS), made of two synchronically rotating birefringent quartz plates, is inserted between the two HWPs to introduce a variable single-photon phase shift $\varphi$ between the two orthogonal polarizations. The input four-photon state of $\left|2_{H}, 2_{V}\right\rangle$ is generated via two pairs of down-converted photons.

In the first experiment, the rotation angle from HWP1 is set to zero so that it has no effect on the $\mathrm{H}$ - and Vpolarizations except for a relative delay but that from HWP2 is set at $\theta=13.7^{\circ}$ so that $\cos ^{2} 2 \theta=(3+\sqrt{3}) / 6$ (angle of polarization rotation is $2 \theta$ ). HWP2 and PBS2 together are equivalent to an asymmetric beam splitter of $T=\cos ^{2} 2 \theta$ and $R=\sin ^{2} 2 \theta$. The fiber coupler for the $\mathrm{H}$-polarized photons is mounted on a micro-translation

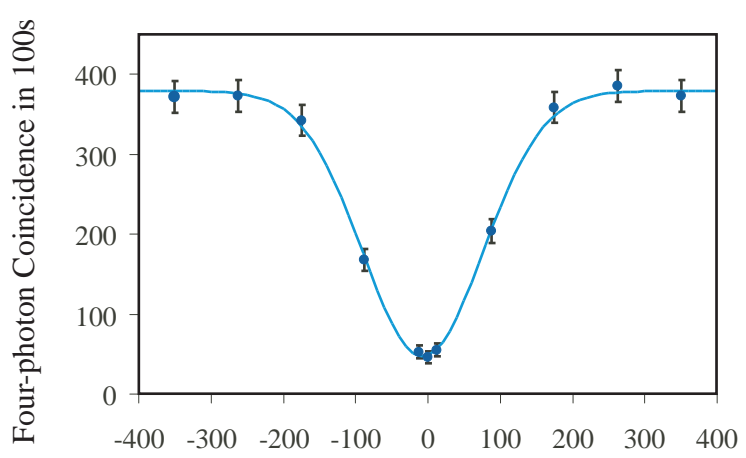

Relative Delay $\Delta(\mu m)$

FIG. 3: Four-photon coincidence as a function of the relative delay $\Delta$ between the $\mathrm{H}$ - and $\mathrm{V}$-polarizations.

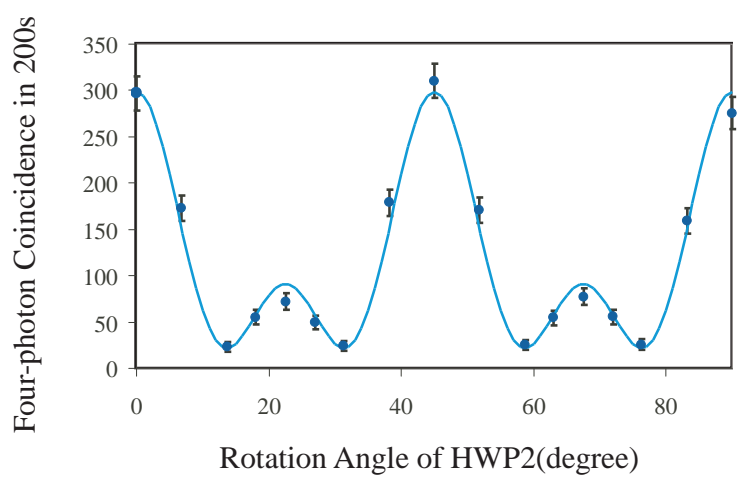

FIG. 4: Four-photon coincidence as a function of the rotation angle of HWP2.

stage to introduce a delay $\Delta$ between the $\mathrm{H}$ - and $\mathrm{V}$ polarizations. Four-photon coincidence counts are registered to measure the probability $P_{4}\left(2_{C}, 2_{D}\right)$ as a function of the delay between the $\mathrm{H}$ - and $\mathrm{V}$-polarizations. The data is shown in Fig. 3 after background subtraction. It shows the typical Hong-Ou-Mandel dip with a visibility of $88 \%$ and a full width at half height of $196 \mu \mathrm{m}$, which are derived from a least square fit to a mathematically convenient Gaussian shape (the solid curve). The less than $100 \%$ visibility is a result of imperfect temporal mode match between the two pairs of down-converted photons $[18,19,20]$. To verify that we indeed have the correct $T$ and $R$ with $\theta$ at $13.7^{\circ}$, we fix the delay $\Delta$ at the bottom of the dip in Fig.3 but change $\theta$. The measured fourphoton coincidence counts after background subtraction are plotted as a function of $\theta$ in Fig.4, which shows four minima at $\theta=13.7^{\circ}, 31.3^{\circ}, 58.7^{\circ}, 76.3^{\circ}$, corresponding to $\cos ^{2} 2 \theta=T=(3 \pm \sqrt{3}) / 6$. Again, the minimum values do not go to zero, due to imperfect temporal mode match. The solid curve is a least square fit to the function [21]

$$
\begin{gathered}
P_{4}(\theta)=C\left[\left(1-1.5 \sin ^{2} 4 \theta\right)^{2}+\left(3 \sin ^{2} 4 \theta-1\right)\right. \\
\left.\times\left(1-\sin ^{2} 4 \theta\right)(1-\mathcal{E} / \mathcal{A}) / 2\right],
\end{gathered}
$$

where $C$ is a scaling factor and $\mathcal{E} / \mathcal{A}$ is a parameter that 


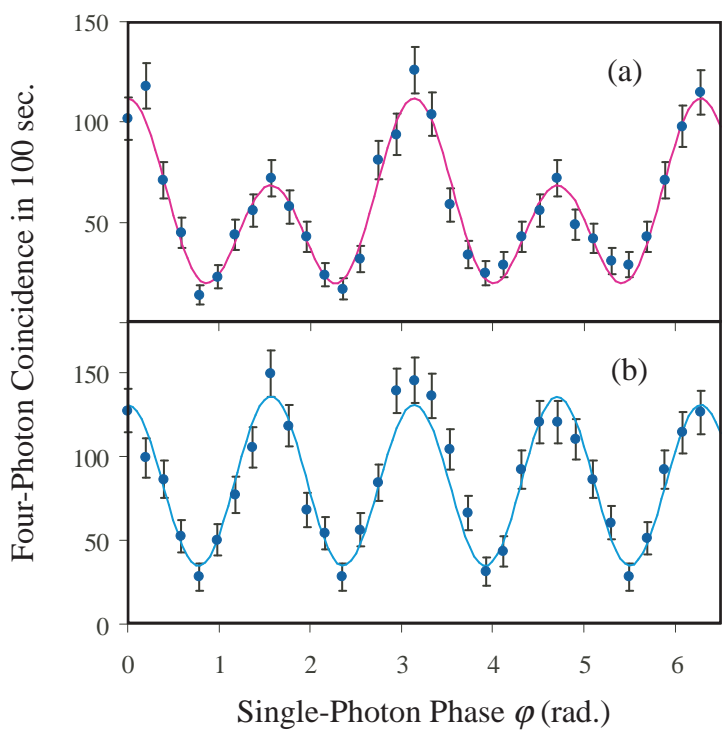

FIG. 5: Four-photon coincidence as a function of the phase difference $\varphi$ between $\mathrm{H}$ - and V-polarizations. HWP1 is set at (a) $\theta=13.7^{\circ}$ and (b) $\theta=13.1^{\circ}$.

characterizes the temporal mismatch [18, 20]. Note that when $\mathcal{E} / \mathcal{A}=1$, the function in Eq.(4) touches zero at the four minima, corresponding to the perfect mode match.

In the next experiment, we set the delay $\Delta$ at the bottom of the dip in Fig. 3 and turn HWP1 to $13.7^{\circ}$ and HWP2 to $22.5^{\circ}$ so that HWP1 serves as the asymmetric beam splitter $(\mathrm{BS} 1)$ with $T=\cos ^{2}\left(2 \times 13.7^{\circ}\right)=(3+$ $\sqrt{3}) / 6$ and HWP2 as the $50: 50$ symmetric beam splitter (BS2) in Fig.1. We measure the four-photon coincidence as a function of the single-photon phase difference $\varphi$ between the $\mathrm{H}$ - and $\mathrm{V}$-polarizations via the phase shifter
(PS in Fig.2). In this way, we form a polarization interferometer equivalent to that in Fig.1. The result of this measurement is shown in Fig.5a. Although it reaches minimum at the values around $\varphi=\pi / 4,3 \pi / 4,5 \pi / 4,7 \pi / 4$, as predicted by Eq.(2), the coincidence has very uneven maxima. This is caused by the imperfect cancellation of the $\left|2_{A}, 2_{B}\right\rangle$ term in Eq.(11) due to temporal mode mismatch 21] as shown in the nonzero minimum in Fig.3. The existence of the $\left|2_{A}, 2_{B}\right\rangle$ term will add a $\cos 2 \varphi$ term to Eq.(2) resulting from interference between $\left|4_{A}, 0_{B}\right\rangle+\left|0_{A}, 4_{B}\right\rangle$ and $\left|2_{A}, 2_{B}\right\rangle$. The data in Fig.5a fits very well to the function

$$
P_{4}\left(2_{C}, 2_{D}\right)=C\left(1+V_{4} \cos 4 \varphi+V_{2} \cos 2 \varphi\right)
$$

with $V_{4}=0.62$ and $V_{2}=0.39$.

Fortunately, the uneven peaks in Fig.5a can be balanced 21] by slightly adjusting HWP1 away from $13.7^{\circ}$ to $13.1^{\circ}$, as shown in Fig.5b. The least square fit for the data in Fig.5b to the function in Eq.(5) gives $V_{4}=0.59$ and $V_{2}=-0.03$. The smallness of $V_{2}$ indicates a good cancellation of the $\cos 2 \varphi$ term in Eq.(5).

In conclusion, we demonstrated both the generalized Hong-Ou-Mandel effect and the de Broglie wavelength of four photons with two pairs of down-converted photons in a scheme involving asymmetric beam splitters. These two effects are a result of four-photon interference.

This work was funded by National Fundamental Research Program of China (2001CB309300), the Innovation funds from Chinese Academy of Sciences, and National Natural Science Foundation of China (Grant No. 60121503). ZYO is supported by the US National Science Foundation under Grant No. 0245421 and No. 0427647.

*Email: zou@iupui.edu
[1] L. Mandel, Rev. Mod. Phys. 71, S274 (1999).

[2] A. Zeilinger, Rev. Mod. Phys. 71, S288 (1999).

[3] D. M. Greenberger, M. A. Horne, and A, Zeilinger, in Bell's Theorem, Quantum Theory, and Conceptions of the Universe, edited by M. Katafos, (Kluwer Academic, Dordrecht, The Netherlands, 1989).

[4] D. Bouwmeester, J. -W. Pan, M. Daniell, H. Weinfurter, and A. Zeilinger, Phys. Rev. Lett. 82, 1345 (1999).

[5] J. J. Bollinger, W. M. Itano, D. J. Wineland, and D. J. Heinzen, Phys. Rev. A 54, R4649 (1996).

[6] Z. Y. Ou, Phys. Rev. A 55, 2598 (1997).

[7] A. N. Boto, P. Kok, D. S. Abrams, S. L. Braunstein, C. P. Williams, and J. P. Dowling, Phys. Rev. Lett. 85, 2733 (2000).

[8] P. Walther, J.-W. Pan, M. Aspelmeyer, R. Ursin, S. Gasparoni, and A. Zeilinger, Nature 429, 158 (2004).

[9] M. W. Mitchell, J. S. Lundeen, and A. M. Steinberg, Nature 429, 161 (2004).

[10] C. K. Hong, Z. Y. Ou, and L. Mandel, Phys. Rev. Lett. 59, 2044 (1987).

[11] Z. Y. Ou, J.-K. Rhee, and L. J. Wang, Phys. Rev. Lett.
83, 959 (1999).

[12] H. Wang and T. Kobayashi, Phys. Rev. A 71, 021802(R) (2005).

[13] K. Sanaka, K. J. Resch, and A. Zeilinger, Phys. Rev. Lett. 96, 083601 (2006).

[14] J. Jacobson, G. Björk, I. Chuang and Y. Yamamoto, Phys. Rev. Lett. 74, 4835 (1995).

[15] B. H. Liu, F. W. Sun, Y. X. Gong, Y. F. Huang, Z. Y. $\mathrm{Ou}$, and G. C. Guo, quant-ph/0610266.

[16] R. A. Campos, B. E. A. Saleh, and M. C. Teich, Phys. Rev. A40, 1371 (1989).

[17] S. Takeuchi, Opt. Lett. 26, 843 (2001).

[18] Z. Y. Ou, J. -K. Rhee, and L. J. Wang, Phys. Rev. A 60, 593 (1999).

[19] K. Tsujino, H. F. Hofmann, S. Takeuchi, and K. Sasaki, Phys. Rev. Lett. 92, 153602 (2004).

[20] Z. Y. Ou, Phys. Rev. A 72, 053814 (2005).

[21] A more detailed multi-mode theory similar to Refs. 18, 20] will take into account the temporal mismatch between the two pairs of down-converted photons. This theory will be presented elsewhere. 